\title{
Latent role of RPL26 in regulating respiratory edema of chronic rhinosinusitis with nasal polyps
}

\author{
Chuanliang Zhao ${ }^{1}$, Jiaxiong Zhang ${ }^{1}$, Wentao Zou ${ }^{1}$, Zimu Zhang ${ }^{2}$, Minlin Jiang $^{2}$, Xiaojing \\ $\mathrm{Cai}^{1}$, and Shaoqing $\mathrm{YU}^{3}$ \\ ${ }^{1}$ Tongji University Tenth People's Hospital \\ ${ }^{2}$ Tongji University \\ ${ }^{3}$ Tongji Hospital, Tongji University, Shanghai, China
}

July 2, 2020

\begin{abstract}
Backgroud: In order to study how to reduce the edema of CRSwNP, we tried to co-cultured CRSwNP with glucocorticoid, predicted and verified possible mechanisms through gene sequencing and CRISPR-Case9 gene editing technology Method:We co-cultured CRSwNP with glucocorticoid, and selected normal CRSwNP as control group. Then analyzed the differentially expressed genes(DEGs), and the RPL26 gene was found through bioinformatics analysis. CRISPR-Case9 was used to establish the RPL26 gene silencing and overexpression cells system; Immunofluorescence staining and flow cytometry analysis were used to observe changes in cell morphology and apoptosis. Results: Pearson Correlation to analyze the correlation of the probe signal values of all specimens and found that the correlation between the two sets of experimental data was high, $\mathrm{R}$ value was close to 1. A total of 12336 genes were detected, and 5026 genes were differentially expressed. Then screened sequence length of [?]1000, 916 of DEGs were obtained, including 340 up-regulated genes and 576 down-regulated genes. Via bioinformatics analysis, the Ribosome pathway is most relevant, and the RPL26 plays an important role. The results of immunofluorescence staining and apoptosis experiments showed that high expression of RPL26 can effectively reduce the degree of edema of CRSwNP cells, and has little effect on apoptosis. Conclusion: Highly expressed RPL26 can effectively reduce the edema of CRSwNP cells, proving that RPL26 plays an important role in this mechanism, and this role of RPL26 may be highly correlated with the ribosome signaling pathway.
\end{abstract}

\section{Introduction}

Chronic rhinosinusitis with nasal polyps(CRSwNP)is a common and frequent disease in otolaryngology. At present, CRSwNP has poor therapeutic and high recurrence rate, and its pathogenesis is not clear. Based on European Position Paper on Rhinosinusitis and Nasal Polyps 2012(EPOS2012) ${ }^{1}$, The treatment of CRSwNP is mainly represented by nasal corticosteroids combined with nasal endoscopic surgery. Although many patients undergo systematic drug-assisted treatment after surgery, they still had pathological features such as nasal mucosa swelling, vesical and mucus discharge. Symptoms such as nasal obstruction, running nose and distention pain persisted or even got worsen. As the research hotspot in the field of nosology, the pathogenesis and recurrence after surgery have attracted much attention ${ }^{2-4}$.

At present, most of the studies on CRSwNP focus on the infiltration of inflammatory cells and expression of cytokine in tissues, but there is still little research on the pathologic changes related to edema and degeneration of nasal mucosal cells in patients with CRSwNP. In recent years, journals such as "Nature" , "Cell" and other magazines had reported eukaryotic cells ribosomal pathway and control system of endoplasmic reticulum-associated protein could regulate polypeptide in cells, and then regulate cellular homeostasis ${ }^{5-8}$. It proved that the mechanism of cell edema induced by hypoxia, infection and other factors was closely 
related to the changes of mitochondrial function. And the factors mentioned above disrupt the metabolism of protein peptides in cells, which in turn leads to cell edema.

Understanding the pathological changes of nasal polyp clearly is important for further studing the pathogenesis of nasal polyp. The pathological changes of tissue cell edema and remodeling in CRSwNP are characterized by intracellular accumulation of abundant collagen and matrix proteins. A large number of inflammatory cytokines are secreted and promote the remodeling of collagen and matrix proteins in the tissues in CRSwNP. Science reported that accumulation of CLCs proteins reshaped airway mucosa and aggravated airway inflammation ${ }^{9}$. Thus it can be seen that accumulation of proteins plays an important role in edema of cells in CRSwNP. Effectively removing the accumulation of proteins in CRSwNP tissues may be a very effective treatment for CRSwNP. Therefore, we designed an experiment to study the differential expression of CRSwNP transcriptome genes in tissues with different degrees of edema treated with glucocorticoids, and predicted the possible mechanism.

\section{Method}

\section{CRSwNP tissue and Cell culture}

This study was approved by the Ethics Committee of the Tenth People's Hospital of Tongji University and the written consent of all specimen providers were obtained. 8 patients with CRSwNP were randomly selected from June 2019 to September 2019 in the otolaryngology department of the Tenth People's Hospital of Tongji University. The diagnosis was based on European Position Paper on Rhinosinusitis and Nasal Polyps 。

Tissue culture in vitro: The nasal polyp tissue sample obtained during the operation was repeatedly washed with PBS solution and cut into tissue pieces of about $5 \mathrm{~mm} 3$ on the clean bench. Then the pieces were disinfected with iodine and digested, then filtered by a sieve, centrifuged, resuspended and placed in a 6 -well plate for culture and passage. Randomly divided into two groups: dexamethasone intervention treatment group (DSTG) group and normal CRSwNP group (CON), 4 for each group.

\section{Transcriptome sequencing}

The Agilent used in this experiment was Mouse lncRNA Microrray V3 (4*180K, Design ID: 084388) . Data analysis were conducted by OE Biotechnology Co., Ltd., (Shanghai, China). the NanoDrop ND2000 (Thermo Scientific) was used to quantifed Total RNA, and the RNA integrity was assessed by Agilent Bioanalyzer 2100 (Agilent Technologies). The sample labeling, microarray hybridization and washing were performed based on the standard protocols. Total RNA were transcribed to double strand cDNA, then synthesized into cRNA and labeled with Cyanine-3-CTP. The labeled cRNAs were hybridized onto the microarray. After washing, the arrays were scanned by the Agilent Scanner G2505C (Agilent Technologies). Feature Extraction software (version10.7.1.1, Agilent Technologies) was used to analyze array images to get raw data. Genespring (version 14.8, Agilent Technologies) were employed to finish the basic analysis with the raw data.

\section{Differentially expressed gene analysis}

To begin with, the raw data was normalized with the quantile algorithm. The probes that at least 1 conditions out of 2 conditions have flags in "P" were chosen for further data analysis. Differentially expressed genes or lncRNAs were then identified through fold change as well as $\mathrm{P}$ value calculated with t-test. The threshold set for up and down-regulated genes was a fold change[?] 2.0 and adj $\mathrm{P}$ value[?] 0.05. Afterwards, GO analysis and KEGG analysis were applied to determine the roles of these DEGs. Finally, Hierarchical Clustering was performed to display the distinguishable genes' expression pattern among samples.

\section{Construction of RPL26 silencing and overexpression cell model}

Both RPL26 silencing and RPL26 overexpression lentivirus transfection plasmids were constructed with the help of the basic medical laboratory of Tongji University Medical School. CRSwNP cells at logarithmic growth stage were randomly divided into three groups, and then performed RPL26 gene silencing and 
overexpression transfection: Group A was transfected with blank plasmid(Control); Group B was transfected with RPL26 silencing plasmid(anti-RPL26); Group B was transfected with RPL26 overexpression plasmid(pre-RPL26). The cells were amplified for 3 days.

\section{Measure the expression of RPL26 protein by Western Blotting}

Part of the samples were digested by trypsin in each group. Cells were collected by centrifugation, and the culture medium was discarded. The cells were washed 3 times with precooled PBS. We added the cells into RIPA cell lysate and incubated for 30min. Protein quantification was performed by western blotting. After electrophoresis, transmembrane, blocking and incubation with anti-Rpl26 Antibody (AB84359) as well as Goat Anti-Rabbit IgG H\&L (HRP) (AB205718), we colored the protein by ECL.

\section{Observe the morphological changes of cells by immunofluorescence staining}

Part of the samples were digested by trypsin in each group and then collected by centrifugation. The cells were adjusted to about $1^{*} 105 / \mathrm{ml}$, dripped onto the glass slide, smeared evenly, and dried at room temperature. The cells were then stained according to the immunofluorescence staining procedure and observed the morphological changes under a microscope. Then we compared the cells with normal nasal polyp tissue .

\section{Cell apoptosis was analyzed by flow cytometry}

Part of the samples were digested by trypsin without EDTA in each group and then collected by centrifugation. After repeated washing with PBS, cell resuspension solution was added for resuscitation, then apoptotic solution (5ul FITC staining solution and 10ul PI staining solution) was added, and the cells were left at 37 darkroom for 10min. After adding cell resuspension solution again, the samples were measured by computer to count the apoptosis rate.

\section{Result}

\section{Original data scanning results and inter-sample correlation analysis}

The original data scan diagram of Agilent chip was mainly used to show the scanning effect of each sample. The chips were dyed with Cy3(green fluorescence). According to the chip detection rate, the lowest detection rate was $58.26 \%$, the maximum detection rate was $66.97 \%$, and the average detection rate was $63.59 \%$. Correlation analysis of all probe signal values using Pearson Correlation found that the two groups of experimental data were highly correlated, and the normalized $R$ value was close to 1(Fig.1B). Subsequently, 126,336 gene signals detected from the two groups of samples were displayed by boxplot (Fig. 1A).

\section{Differential multiple of probe gene and volcano plot distribution}

In the group comparison with biological repeats, the differential multiple and $p$ value were usually used to determine the DEGs. Volcano Plot could be used to display the distribution of difference multiples and P value clearly at the same time, and finally screened the distribution of the different genes (Fig.2).

\section{Analysis of differentially expressed genes}

We analyzed the expression data of CON and DSTG, and a total of 28425 DEGs were detected. We set the filter condition $|\log 2 \mathrm{FC}|[?] 2$, adjP Value[?]0.05, and a total of 5062 differentially expressed genes were selected. After screening again with length[?]1000 pairs, there were a total of 916 DEGs left, including 340 up- regulated genes and 576 down-regulated genes(Table. 1).

\section{GO enrichment analysis and PPI network analysis}

A total of 916 DEGs mentioned above were analyzed by using the DAVID online tool. GO enrichment analyzed the result from biological processes, cell component and molecular function(Fig.3A). At the same time, the protein-protein interaction(PPI) network helped us systematically investigate the mechanism of the DEGs in the complete transcriptome of nasal polyp cells. A total of 916 DEGs were analyzed using the 
STRING online tool. 916 genes were introduced into PPI network, and then the PPI network was analyzed through MCODE in Cytoscape. Finally, a total of 2 core protein interaction networks were identified(Fig.3B).

\section{KEGG analysis and signal pathway prediction of core gene in PPI network}

The PPI network was analyzed by Cytoscape tool. The STRING network score was set to be greater than three. A total of DEGs including all genes in the two core networks were obtained. We performed KEGG functional analysis on the DEGs again, and the results showed that the DEGs were mainly shown in the Ribosome pathway(Fig.3C).

\section{RPL26 gene annotation and verifying differential expression in CRSwNP by PCR}

Through functional analysis of the core network as well as ribosome signaling pathway anchoring genes and NCBI database annotation we found that all the 15 key genes in the ribosome signaling pathway were included in the 16 core network interacting genes. There were 3 up-regulated genes and 13 down-regulated genes(Fig.3C). Through NCBI database and GenCards website notes, we found that RPL26 gene played an important role in this process. To verify whether RPL26 was differentially expressed in CRSwNP, we cultured nasal polyp tissue cells in vitro again. Normal nasal mucosa group (Control), nasal polyp group $(\mathrm{CON})$ and dexamethasone intervention group (DTSG) were cultured for 3 days. All the cells were collected for quantitative PCR analysis. The results indicated that the RPL26 gene expression in nasal polyp tissues was lower normal nasal mucosa, but after dexamethasone intervention, the RPL26 gene expression level was significantly increased(Fig.4).

\section{Establishing of RPL26 silencing and RPL26 overexpression cells}

After RPL26 silencing and overexpression models were established, the expression of RPL26 protein was quantitatively detected by BCA method. The results showed that, compared with the Control group, the expression level of RPL26 protein in the anti-RPL26 group was significantly decreased, while the expression level of RPL26 protein in the pre-RPL26 group was increased, indicating that RPL26 silencing and overexpression cells were transfected successfully(Fig.5A).

\section{Observe the morphological changes of cells by immunofluorescence staining}

We selected some of the cells and stained the cells with immunofluorescence staining. Obvious We selected some cells and stained them with immunofluorescence staining. Observe the morphological changes of cells in each group: compared with the CON group, the interstitial cells in the anti-RPL26 group had too much edema, while the edema in the pre-RPL26 group was significantly reduced, and the anti-RPL26 group was more obvious than the pre-RPL26 group (Fig.5 B, C, D). This result indicates that the high expression of RPL26 gene can effectively reduce the degree of cell edema.

\section{Detecting the apoptosis effect of RPL26 on CRSwNP cells by flow cytometry}

We further examined the apoptotic effect of RPL26 on CRSwNP cells. Flow cytometry was used to detect the Control group, anti-RPL26 group and pre-RPL26 group. The results showed that there was no significant difference in apoptosis rate among the three groups; Compared with the pre-RPL26 group, the apoptosis rate of the anti-RPL26 group was slightly higher, but there was no statistical significance(Fig.6).

\section{Discussion}

With the development of experimental technology, especially electron microscopy, it is known that in patients with CRSwNP, the patient's nasal mucosal epithelial cells are edema degeneration, the barrier function of the nasal mucosa is impaired, and the resistance to harmful substances and microorganisms is insufficient, which leads to repeated inflammatory reactions in the nasal cavity, and then gradually lead to the remodeling of nasal mucosa. Transmission electron microscope observation showed that the CRSwNP tissue showed edema of nasal mucosa epithelial cells, a large number of mitochondrial hyperplasia in the cytoplasm, endoplasmic reticulum edema and the formation of a large number of swallowed vesicles; Submembrane edema, dilation of small blood vessels and capillaries in the tissue, intrinsic interstitial edema accompanied by a large number of 
inflammatory cells ${ }^{10-13}$. Also in clinical diagnosis, the most common pathological classification of CRSwNP disease is edema polyps, accounting for about $85 \%-90 \%$.

In addition to functional endoscopic surgical resection, glucocorticoids are widely used in clinical treatment of CRSwNP, and their therapeutic effect has reached consensus. Local and systemic application of glucocorticoids in the nasal cavity is considered to be effective for the treatment of CRSwNP. Cochrane evaluation showed that glucocorticoids can effectively improve systemic symptoms of patients with nasal polyps and reduce edema of nasal polyps. This mechanism is proved to be that glucocorticoid can quickly enter the cell nucleus and interact with the glucocorticoid response element on the chromosome by binding to its receptor, initiating or inhibiting downstream gene expression, thereby exerting a physiological role. However, although there is a clear understanding of the structural changes of CRSwNP tissues and cells, the mechanism of the formation of CRSwNP tissue edema is still unclear.

According to the mechanism of glucocorticoid treatment of CRSwNP, we tried to co-cultured CRSwNP cells with a glucocorticoid-rich medium to observe the changes in edmea of CRSwNP, and then analyze the expression of gene transcriptomes to compare with normal cultured CRSwNP cells. Then we analyzed the differentially expressed genes of the two groups, so as to predict the mechanism of nasal polyp edema is related to that signal, while using CRISPR-Case9 gene editing technology for simple verification.

In immunofluorescence staining of CRSwNP tissues, we found that a large number of interstitial cells edema increased, a large number of inflammatory cells infiltration. After the intervention of glucocorticoid, the edema of CRSwNP cells is significantly reduced by the difference between the CON group and the DSTG group. GO enrichment, KEGG analysis and PPI network analysis revealed that this mechanism is closely related to the ribosome pathway involved in RPL26. This proves that the RPL26 gene may play an important physiological role in the mechanism of CRSwNP cells edema.

Cell Research reported that RPL26 is the main substrate for ubiquitination of mammalian cells and a special regulator of ubiquitination of ribosomal proteins ${ }^{5}$. The RPL26 gene encodes the ribosomal protein of the L24P family and is highly expressed in the mitochondria in the cytoplasm, which can regulate mitochondrial function. RPL26 encodes a protein that can effectively eliminate ectopic stasis in the cell after ubiquitination, and this effect is not through the traditional eukaryotic endoplasmic reticulum-related protein degradation protein quality control system (ERAD) or cytosolic ribosome The quality control (RQC) system works, but transports the accumulated polypeptide from the endoplasmic reticulum to the lysosome in the cytoplasm for degradation ${ }^{14,15,16}$.

We established RPL26 silencing and overexpressing CRSwNP cell system. The study found that after RPL26 silencing expression, the degree of CRSwNP tissue edema increased; but after RPL26 overexpression, CRSwNP edema was significantly reduced. In the apoptosis experiment, no matter whether it is RPL26 silencing system or RPL26 over-expression, there is no significant difference in apoptosis between the two groups of experiments and normal CRSwNP tissue. Highly expressed rpl26 can effectively reduce the degree of cell edema and have little effect on apoptosis. The higher apoptosis rate was detected in anti-rpl26 group, which may be related to excessive cell edema. This result is consistent with the results of pretranscriptome genetic analysis, confirming that RPL26 plays an important role in the regulation of CRSwNP tissue edema. Using online gene annotation sites and ncbi database to query information, the function of RPL26 may be completed through the ribosomal signaling pathway ${ }^{17,18,19}$, but this conjecture still needs further experimental confirmation.

\section{Concluision}

In summary, this study intervened the growth of CRSwNP tissues by glucocorticoids, analyzed the differential gene expression of transcriptomes from normal CRSwNP tissues, and initially predicted that the RPL26 gene played an important role in the process of edema of CRSwNP tissues. Then we established the RPL26 silencing and overexpressing CRSwNP cell system through CRISPR-Case9 gene editing technology, and studied the effect of RPL26 silencing and overexpression on CRSwNP tissue edema. It was initially verified that the high expression of RPL26 gene can reduce the degree of CRSwNP tissue edema, proving that RPL26 
gene plays an important role in the regulation of CRSwNP tissue edema. However, the mechanism by which the rpl26 gene functions still needs further research and confirmation.

\section{Ethical disclosures Confidentiality of data}

The authors declare that no patient data appears in this article. Right to privacy and informed consent. The authors declare that no patient data appears in this article. Protection of human subjects and animals in research. The authors declare that no experiments were performed on humans or animals for this investigation.

\section{Founding}

This work was founded by the National Science Foundation of China (No. 8187040043); Western Medicine Guide Project of Shanghai City (No. 17411970500); Shenkang Medical Development Center Clinical Science and Technology Innovation Project of Shanghai City (No. SHDC 12019X07); Health Commission Advanced Technology Promotion Project of Shanghai City (No. 2019SY071); the research funding of tenth people's hospital of Tongji University (No. SYGZRPY2017035).

\section{Conflicts of interest}

The authors report no conflicts of interest. The authors alone are responsible for the content and writing of this manuscript.

\section{Reference:}

1. Fokkens WJ, Lund VJ, Mullol J, et al. EPOS 2012: European position paper on rhinosinusitis and nasal polyps 2012[J]. A summary for otorhinolaryngologists. Rhinology, 2012, 50(1): 1-12.

2. Shi JB, Fu QL, Zhang H, et al. Epidemiology of chronic rhinosinusitis: results from a cross-sectional survey in seven Chinese cities[J]. Allergy, 2015, 70(5): 533-539.

3. Dykewicz MS, Hamilos DL. Rhinitis and sinusitis[J]. J Allergy Clin Immunol, 2010, 125(2 Suppl 2): S103-S115.

4. Smith KA, Pulsipher A, Gabrielsen DA, et al. Biologics in Chronic Rhinosinusitis: An Update and Thoughts for Future Directions[J]. Am J Rhinol Allergy, 2018, 32(5): 412-423.

5. Wang Lihui, Xu Yue,Rogers Heather, Saidi Layla, et al. UFMylation of RPL26 links translocationassociated quality control to endoplasmic reticulum protein homeostasis.[J]. Cell research, 2020, 30(1)

6. Walczak C, Leto D, Zhang L, et al. Ribosomal protein RPL26 is the principal target of UFMylation. $[\mathrm{J}]$. Proceedings of the National Academy of Sciences of the United States of America, 2019, 116(4).

7. Deniz S, Gerald C, Ryan A, et al. The Mammalian Ribo-interactome Reveals Ribosome Functional Diversity and Heterogeneity[J]. Cell, 2017, 169(6).

8. Garreau D, Prokhorova I, Rodnina M, et al. Structural basis for the inhibition of the eukaryotic ribosome.[J]. Nature, 2014, 513(7519).

9. Person EK,Verstraete K,Heyndrickx I,et al. Protein crystalization promotes type 2immunity and is reversible by antibody treatment[J]. Science, 2019, 364 (6442):751-765.

10. Nakayama T, Asaka D, Yoshikawa M, et al. Identification of chronic rhinosinusitis phenotypes using cluster analysis[J]. Am J Rhinol Allergy, 2012, 26(3): 172-176.

11. Soler ZM, Hyer JM, Ramakrishnan V,et al. Identification of chronic rhinosinusitis phenotypes using cluster analysis[J]. Int Forum Allergy Rhinol, 2015, 5(5): 399-407.

12. Lou H, Meng Y, Piao Y, et al. Cellular phenotyping of chronic rhinosinusitis with nasal polyps[J]. Rhinology, 2016, 54(2): 150-159.

13. Wang X, Zhang N, Bo M, et al. Diversity of TH cytokine profiles in patients with chronic rhinosinusitis: A multicenter study in Europe, Asia, and Oceania[J]. J Allergy Clin Immunol, 2016, 138(5): 1344-1353.

14. Joazeiro CAP. Ribosomal Stalling During Translation: Providing Substrates for Ribosome-Associated Protein Quality Control. Annu Rev Cell Dev Biol. 2017;33:343-368. doi:10.1146/annurev-cellbio111315-125249. 
15. Brandman O, Hegde RS. Ribosome-associated protein quality control. Nat Struct Mol Biol. 2016;23(1):7-15. doi:10.1038/nsmb.3147.

16. Simsek D, Tiu GC, Flynn RA, et al. The Mammalian Ribo-interactome Reveals Ribosome Functional Diversity and Heterogeneity. Cell. 2017; 169(6): 1051-1065. e18. doi: 10. 1016/j. cell. 2017.05.022.

17. Brandman O, Hegde RS. Ribosome-associated protein quality control. Nat Struct Mol Biol. 2016;23(1):7-15. doi:10.1038/nsmb.3147.

18. Juszkiewicz S, Hegde RS. Initiation of Quality Control during Poly(A) Translation Requires Site-Specific Ribosome Ubiquitination. Mol Cell. 2017; 65(4): 743-750. e4. doi: 10.1016/j.molcel.2016.11.039.

19. Shao S, Hegde RS. Reconstitution of a minimal ribosome-associated ubiquitination pathway with purified factors. Mol Cell. 2014;55(6):880-890. doi:10.1016/j.molcel.2014.07.006.

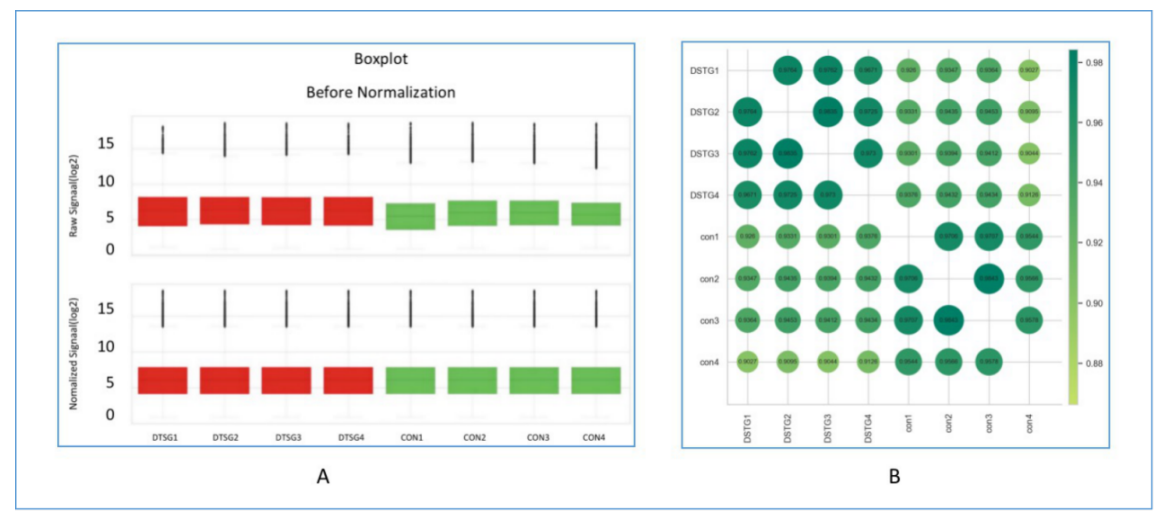

Fig. 1: A: Box plot showing 126,336 gene signals detected from the sample sample. B: Pearson Correlation for correlation analysis of samples. $\mathrm{R}$ value was close to 1 , proved that the experimental design was reasonable and the reliability of the results was extremely high.

Table 1: Detection of total mRNA and differential gene screening results.

\begin{tabular}{|c|c|}
\hline & mRNA \\
\hline Total DEGs & 28425 \\
\hline Filtered DEGs & 5062 \\
\hline Lenth $\leq 1000$ & 916 \\
\hline Up-regulated & 340 \\
\hline Down-regulated & 576 \\
\hline
\end{tabular}




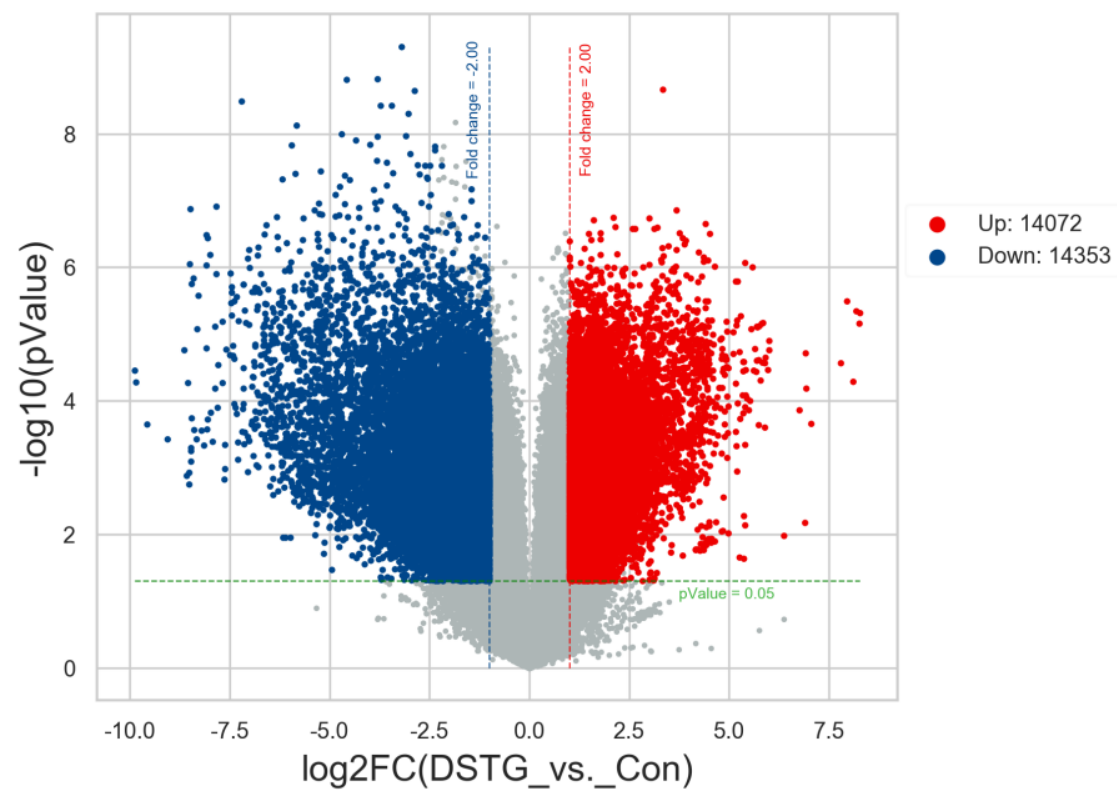

Fig. 2: Screen according to $|\log 2 \mathrm{FC}|[?] 2$ and p[?]0.05. Points represent genes, red dots indicate up-regulation, blue dots indicate down-regulation, and gray areas indicate non-differentially expressed genes.
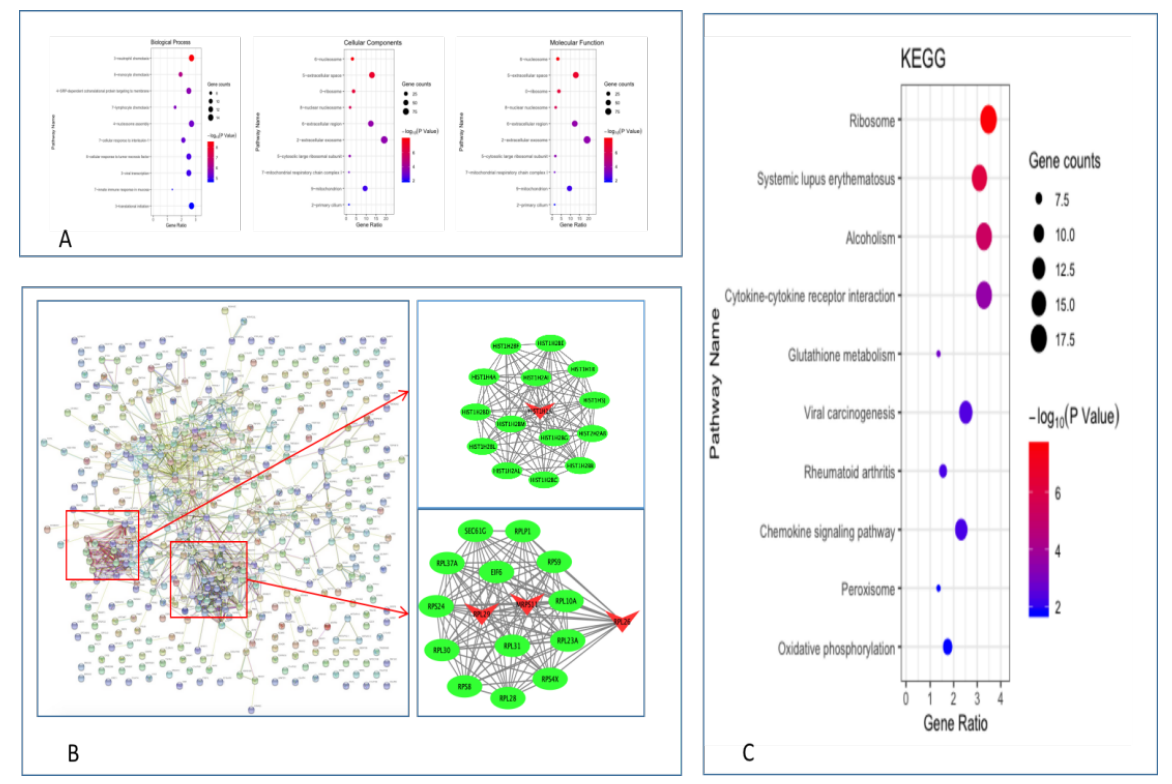

Fig. 3: A: Gene ontology analysis of the 916 differentially expressed genes. The bubble chart shows the results of the enrichment analysis of BP, CC and MF. The size of the bubble represents the number of genes, and the color from blue to red indicates the significance. B: PPI network constructed by STRING online tool and analyze the core module.there were a total of 916 genes in the PPI network complex, the nodes meant proteins, the edges meant interaction. Through the STRING network score setting greater than 3, Cytoscape analysis found two core networks . C: KEGG pathway analysis of the DEGs. The size of the bubble represents the number of genes, and the color from blue to red indicates the significance. 


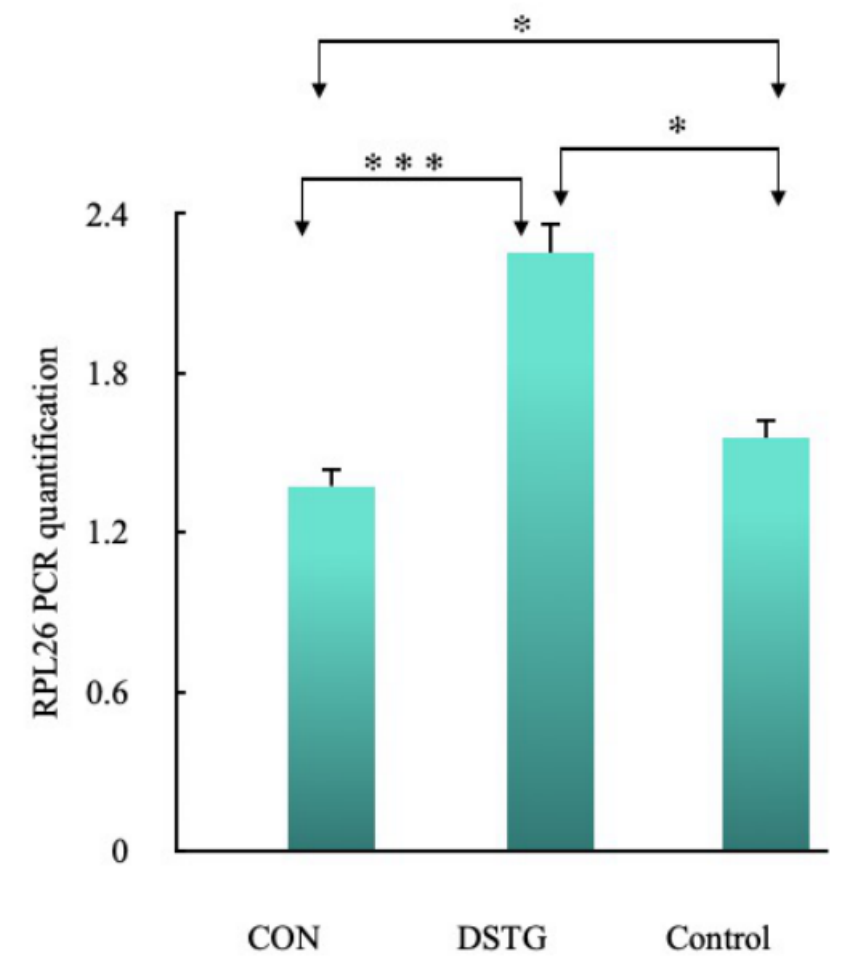

Fig. 4: The results of quantitative PCR analysis indicated that the expression of RPL26 in CRSwNP tissues decreased, but after dexamethasone intervention treatment, the expression of RPL26 increased significantly.
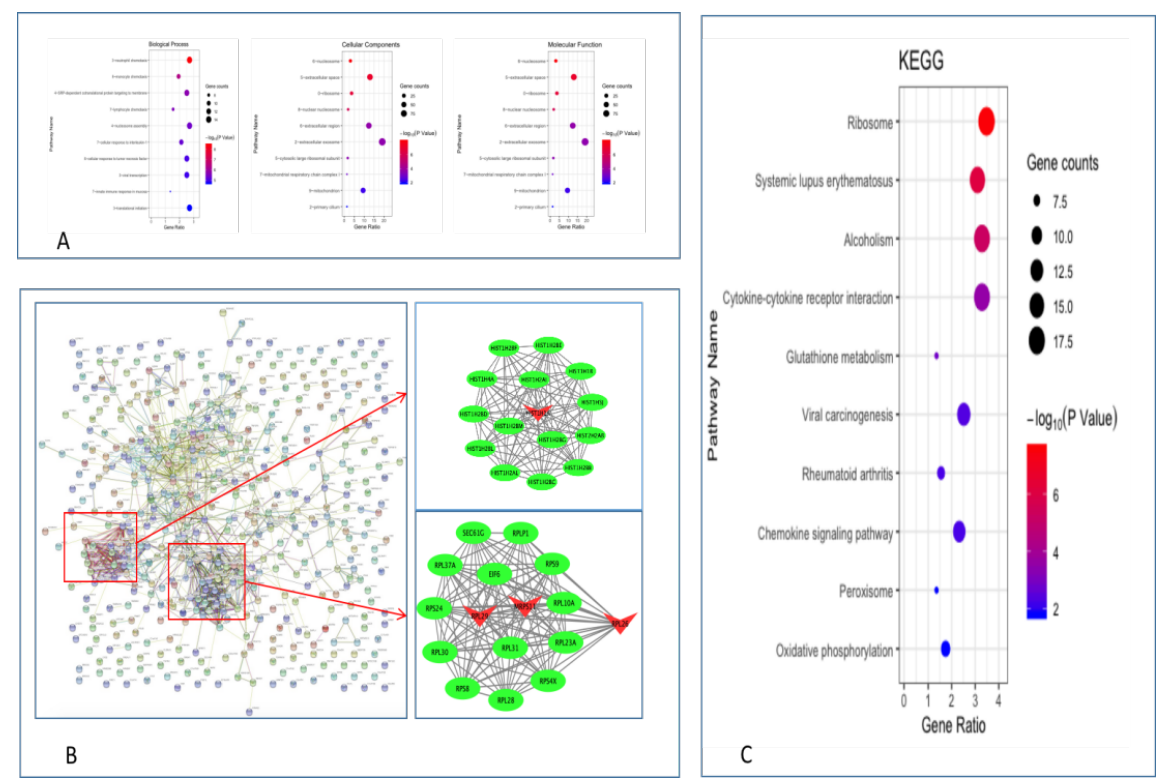

Fig. 5: A: CON: normal CRSwNP cells group; pre-RPL26: RPL26 overexpression group; anti-RPL26: 
RPL26 silencing group. The results of WB suggest that the RPL26 protein in the pre-RPL26 group is highly expressed, but the RPL26 protein in the anti-RPL26 group is basically not expressed. B: Immunofluorescence staining showed that the cell edema in the pre-RPL26 group was significantly reduced compared with the con group, while the cell edema in the anti-RPL26 group increased.
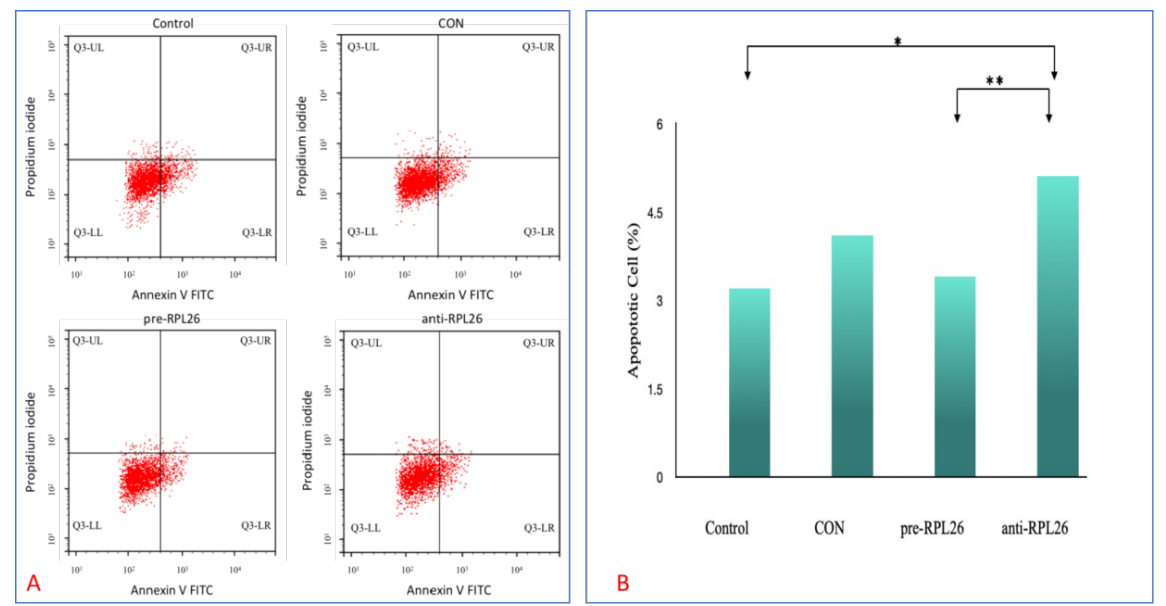

Fig. 6: Apoptosis experiments showed that the anti-RPL26 group increased apoptosis compared with normal nasal mucosa (Control) and pre-RPL26 group, and this result may be related to excessive cell edema. The other groups have no difference in the rate of apoptosis. 